\title{
Rheumatic Heart Disease Classification Using Adaptive Filters
}

\author{
Nanda Kishor Panda ${ }^{1}$, Monica Subashini M. ${ }^{2 *}$, and Milind Kejriwal ${ }^{3}$ \\ ${ }^{1}$ Student member, IEEE \\ ${ }^{2}$ School of Electrical Engineering, Vellore Institute of Technology, Vellore \\ ${ }^{3}$ School of Electronics Engineering, Vellore Institute of Technology, Vellore
}

\begin{abstract}
An efficient and innovative method has been proposed in this paper to detect heart murmurs as a method to identify rheumatic fever with the use of adaptive filters, transform techniques and Neural Network Algorithms by considering various parameters such as number of peaks, Signal to Noise Ratio (SNR) and Power Spectral Density. Under optimum conditions the classification returned exact outputs even when the neural network was trained under false positive data thus showing its effectiveness.
\end{abstract}

\section{Introduction}

Health care solutions have seen a tremendous growth in recent years. With improving technologies, the field of health care diagnosis relies heavily on sophisticated equipment, precise sensors and optimized processing of signals to support the traditional experience of medical practitioners. Yet there is a constant requirement to improve these factors. Auscultation has been one of the oldest and most common diagnostic tools for a variety of symptoms.

Recent trends have significantly improved this process through computer aided auscultation by reducing false negative readings for heart murmurs from $13.3 \%$ to $7.1 \%$ as shown by Raymond [1]. Reducing signal noises due to environment and system is one of the most challenging aspects of computer aided detection due to the extremely low power of the signal requiring very high sensitivity of equipment. There are various methods by which this can be achieved for various signals. Mohammad (et. al) proposed a method for noise reduction in coronary artery disease detection which broadly involves spectral analysis and segmentation to identify noisy frames and then using adaptive filters to remove the noise [2]. Neural networks have been shown to improve the performance of such methods in transformation and feature extraction with improved algorithms as shown by Dandan (et al) [3]. Kalman filtering is a widely-used technique for a variety of applications. It's a robust technique which is used to remove statistical noise by conducting successive measurements over time and estimates the error. It finds application in camera based photo plethysmography [4] which is a noninvasive technique used to measure heart rate.

\footnotetext{
*e-mail: monicasubashini.m@gmail.com
} 
Transformation of real time signals is an important stage as features of the signal which are useful are not easily identifiable in time domain. A few common transformation techniques for bio-medical signals include discrete S-transform [5], time-frequency analysis [6], [7] and Hilbert-Huang Transform [8]. Upon transformation to the required domain, the required features of the signal need to be extracted. Power Spectral Density (PSD), Energy Envelope [9], peak location [10], are some of the most common features considered for heart signals.

In this paper, neural networks have been proposed to detect heart murmurs by identifying peaks as an early warning system for rheumatic fever. Among various commonly used neural networks scaled conjugate gradient back propagation algorithm has been shown to be the most adept at handling the varying data set.

\section{Background Information}

\subsection{Rheumatic heart diseases}

Rheumatic fever is an inflammatory disease caused in small children in the age group 0-5 yrs. This is most predominant in developing nations like India, China, etc. This can cause permanent damage to heart valves if left undetected and not treated timely. The main cause of heart damage is due to prolonged rheumatic fever causing inflammation of heart.

\subsection{Diagnosis}

Due to the fact that this disease occurs mostly in children of developing countries early detection of heart abnormalities are hardly accessible, resulting in premature deaths, paralysis and permanent damage to brain. ECG is most effective way to detect these abnormalities but needs special instruments and trained technicians. This paper presents an new approach of efficient and accurate detection of heart abnormalities using phonocardiogram.

\subsubsection{The Phonocardiogram}

The Phonocardiogram (PCG) is simply the audio signal of heart sound, which is caused due to the rhythmic contraction and expansion of heart muscles [11]

As shown in Fig. 1 a PCG signal can be divided into 2 major sections as S1 and S2. The S11-heart cycle is further divided into 4 major components: sound due to the closure of the mitral and tricuspid valves (S1), systolic period (S12), sound corresponding to the closure of the aortic and pulmonary valves (S2), and the diastolic period (S21). With correct identification of different sections of PCG heart valve abnormalities which are the major side effects of Rheumatic fever can be accurately determined [12]-[14].

\section{Proposed Topology}

A screening procedure for detection of heart abnormalities due to rheumatic fever using signal processing and machine learning was developed. The phonocardiogram of affected people contains a lot of extra sounds along with the regular systole and diastole peaks. Detection of these extra peaks can be considered as an early indication for people at risk. The steps involved in achieving this are filtering, transformation, feature extraction and classification performed using a variety of methods to obtain the optimal result. The proposed process flow is as shown in Fig. 2. 

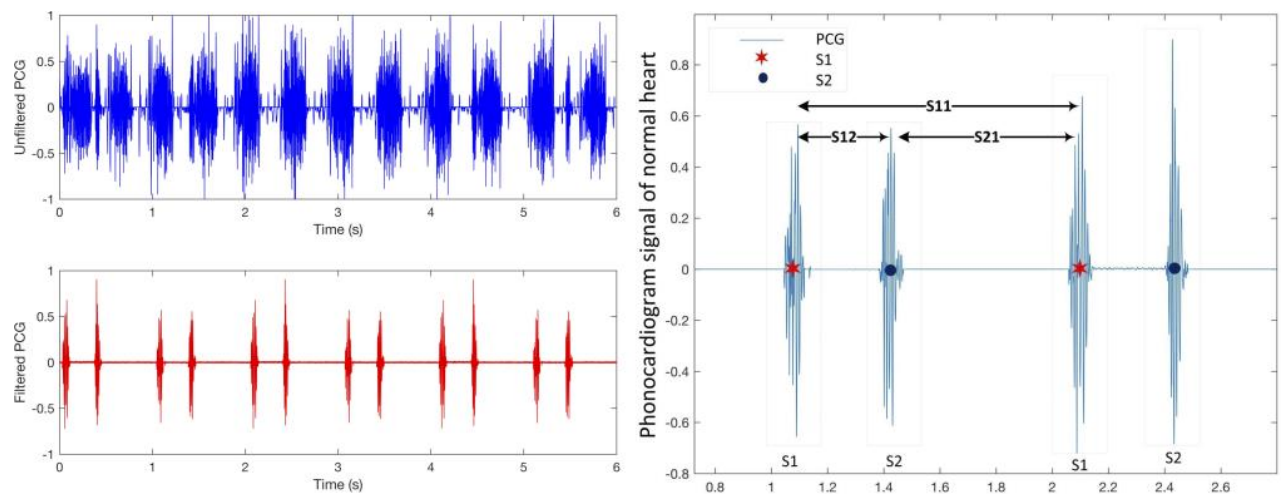

Fig. 1. Sections of phonocardiogram (PCG), with components: first Sound (S1), second sound (S2), systolic (S12), diastolic (S21) and heart cycle (S11) periods (Left). The Phonocardiogram of a Normal Heart (Right).

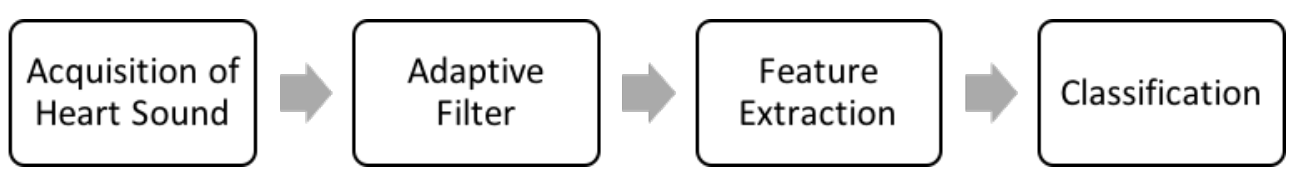

Fig. 2. Process flow for detection of heart abnormalities.

\subsection{Signal Acquisition}

The heart sound data base used for training and validation has been obtained from University of Michigan heart Sound and Murmur Library. The obtained data base had two sets one from digital stethoscope and the other from a mobile app. Each set comprised of normal, artifacts, extrahls, murmurs, unlabeled heart sounds. Further a second public* database was used for testing and validating the results. The sounds in this data set was recorded using a digital stethoscope DigiScope ${ }^{\circledR}$ during clinical trials.

\subsection{Adaptive Filtering}

The filtering of the signal is essential due to the inherent noise in systems. A digital adaptive filter is applied to the noisy signal to obtain the pure signal as shown in Fig. 3.

The adaptive filter used in filtering is as shown in Fig. 3. Starting point of the process is the unfiltered PCG, $x[n]$.

$$
x[n]=P C G+\text { noise }(1)
$$

A reference signal which is correlated with $d[n]$ and uncorrelated with $x[n]$, is used to eliminate the interference. The major components of noise in the PCG signal are: $50 \mathrm{~Hz}$ power supply hums, lung noises, gastrointestinal noises, noise due to breathing. In order to suppress the above noises a signal $\mathrm{d}[\mathrm{n}]$ is gripped at the power supply and from other known noisy signals. It has the same frequency, but different amplitude and phase compared to noise[n]. Unlike frequency-selective filters the adaptive filter is applied to $x[n]$ instead of the primary 


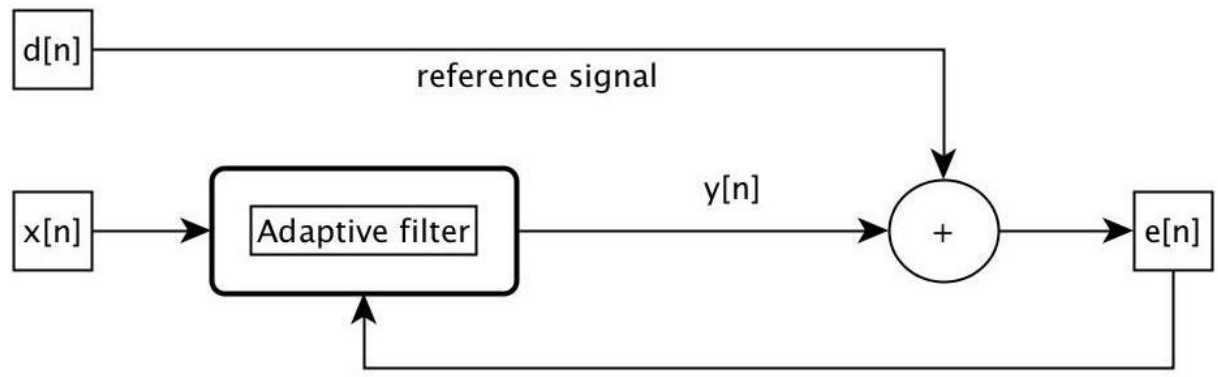

Fig. 3. Block Diagram for Adaptive Filtering.

input $d[n]$. The filter output $y[n]$ is an estimate of noise[n]. Subtracting this from the underlying signal $x[n]$ we get the error signal e[n], an estimate of PCG[n]. Adaptive filters are preferably FIR which are stable and have simple cost function as shown below:

$$
J=E\left\{e^{2}[n]\right\}=(d[n]-y[n])^{2} \rightarrow \min .
$$

For a $\mathrm{N}$ order filter the above equation results in a quadratic cost-function with a global minimum. $\mathrm{R}$ is the auto correlation matrix of $\mathrm{x}[\mathrm{n}]$ and $\mathrm{P}$ is the cross-correlation-vector between $\mathrm{x}[\mathrm{n}]$ and $\mathrm{d}[\mathrm{n}]$.

$$
J=E\left\{e^{2}[n]\right\}=E\left\{d^{2}[n]\right\}-2 W^{T} P+W^{T} R W
$$

Where, $\mathrm{W}=\left(\mathrm{W} 0, \mathrm{~W} 1, \ldots, \mathrm{W}_{N-1}\right)$ and $\mathrm{T}=$ Filter Coefficients. using:

For each iteration step the filter coefficients for the upcoming step $\mathrm{W}(\mathrm{n}+1)$ are computed

$$
W(n+1)=W(n)+\mu e[n] * x(n)
$$

For guaranteed convergence for LMS-algorithm, $\mu$ taken as the maximal amplitude of the reference signal $(\max (\mathrm{d}[\mathrm{n}]))$.

The upper bound is defined as:

$$
\mu_{\max }=\frac{2}{3 * N * \max (x)^{2}}
$$

The LMS-algorithm only converges to a good approximation. To measure the deviation of approximation from exact solution a miss-adjustment $M$ is introduced. It depends on the average power of the reference signal and the step-size $\mu$.

$$
M=\frac{\mu}{2} * N * \operatorname{power}(x)
$$

For quick convergence, a small step size is essential, but a small step-size leads to a large convergence time. The convergence time $\tau$ can be expressed as:

$$
\zeta \approx \frac{1}{4 \alpha} * \kappa(R)
$$


where, $\alpha$ is the applied step-size normalized to the maximum possible $\mu_{\max } . \lambda_{\max }$ and $\lambda_{\min }$ are the largest and smallest eigenvalue of $\mathrm{R}$ respectively. Also, $\mathrm{k}(\mathrm{R})$ is given by:

$$
\kappa(R)=\frac{\lambda_{\max }}{\lambda_{\min }} * \text { condition number }
$$

The proposed LMS-algorithm shows slow convergence for non-smooth signals and fast convergence for uniformly distributed spectrum signals. Here the filter order is set as: $\mathrm{N}=4$. The unfiltered PCG signal and filtered PCG signal is as shown in Fig: 3.

\subsection{Feature Extraction}

The number of such peaks for the entire duration of the sample is obtained and taken as a parameter for training the neural network. The SNR of the signal is also included as a parameter.

$$
\text { Shannon energy is given by: } E=-x^{2} \log \left(x^{2}\right)
$$

Apart from the above parameters the following parameters were extracted for classification as shown in Table: 1. The peaks detected were as shown in Fig: 4.
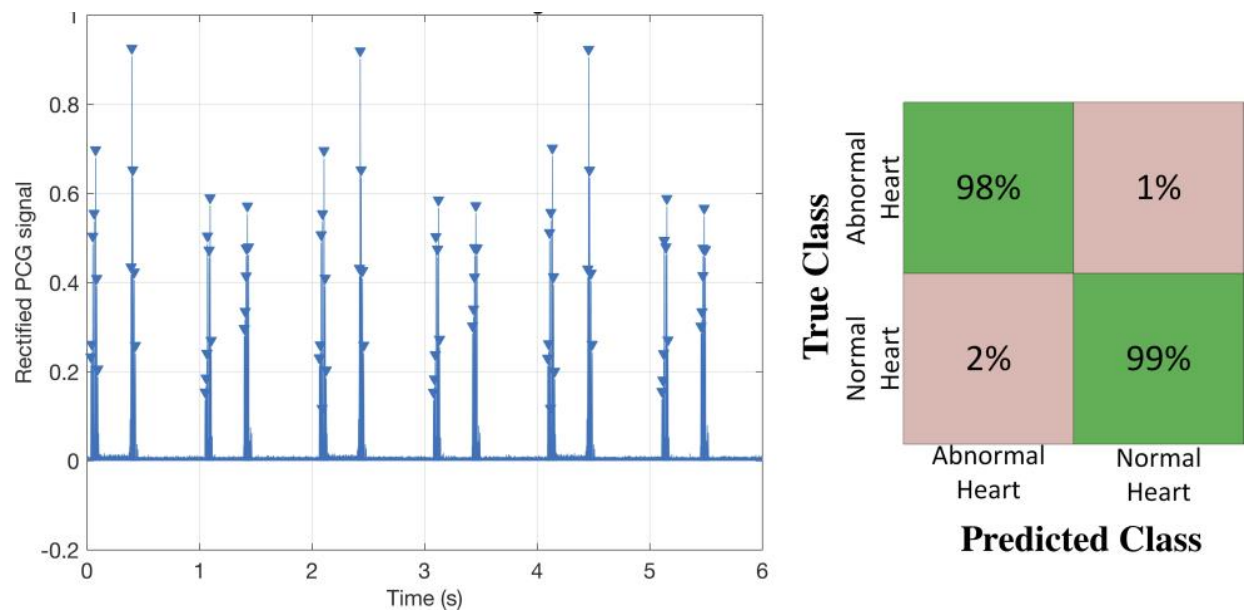

Predicted Class

Figure 4. Peak detection of rectified PCG signal (Left). Confusion Matrix validating the accuracy of training, with target class named as normal and abnormal(Right).

Table 1. List of features extracted.

\begin{tabular}{cccc}
\hline SI. No: & Time domain & Statistical domain & Frequency domain \\
1 & Total power & Mean & Peak Frequency \\
2 & Peak Amplitude & Standard Deviation & Intermodulation Distortion \\
3 & Zero Crossing rate & Variances & Total harmonic Distortion \\
4 & RMS & Skewness & Dynamic Range \\
\hline
\end{tabular}




\subsection{Classification}

The extracted parameters are tabulated for the entire sound bank and fed into various algorithms as describes in Table: 2. For each of the algorithm [15] the performance is compared with and without the use of adaptive filter. When a heart sound with abnormally large number of peaks or a relatively low SNR is given as an input to the algorithm, the system classifies it as an abnormal sound and thus indicates that the concerned person has a higher chance of being affected by heart abnormalities.

\section{Results and discussions}

A new way of effective classification of different heart abnormalities caused due to Rheumatic fever was shown in this paper. The raw signal acquired from different sources were effectively filtered as shown in Fig: 1. Further a number of, time domain, frequency domain and statistical features were extracted from the PCG signal. One such feature of peak detection is shown in Fig: 4. The extracted features were used to train a Neural network. The results of the training were satisfactory and is shown in Fig: 4.

Table 2. Comparison of various algorithms.

\begin{tabular}{|c|c|c|c|c|c|c|c|c|c|}
\hline \multicolumn{2}{|c|}{ Algorithm } & \multicolumn{3}{|c|}{ Decision trees } & \multicolumn{4}{c|}{ KNN } & SVM \\
\hline \multicolumn{2}{|c|}{ Type } & Complex & Medium & Simple & Fine & Coarse & Cubic & Weighted & Linear \\
\hline \multirow{3}{*}{} & $\begin{array}{c}\text { Without } \\
\text { adaptive } \\
\text { filter }\end{array}$ & 80.4 & 76.0 & 84.2 & 90.3 & 90.0 & 77.0 & 92.4 & 93.2 \\
\cline { 2 - 9 } & $\begin{array}{c}\text { With } \\
\text { adaptive } \\
\text { filter }\end{array}$ & 83.8 & 83.8 & 85.2 & 94.0 & 94.0 & 77.0 & 95.4 & 96.8 \\
\hline
\end{tabular}

\section{Future scope}

The whole procedure introduced in this paper can be effectively used in the screening for heart abnormalities in rural areas. In most rural areas people lack awareness about the different heart diseases. A smart stethoscope can be integrated with a Digital Signal Processor and it than can be used for dynamic assessing of the heart valve abnormalities. This can be used in telemedicine purposes also.

\section{References}

1. R. L. Watrous, Annu. Int. Conf. IEEE Eng. Med. Biol. Proc pp. 140-143 (2006)

2. Proc. Annu. Int. Conf. IEEE Eng. Med. Biol. Soc. EMBS pp. 5880-5883 (2011)

3. Y. L D. Song, L. Tao, Icsai pp. 1677-1681 (2012)

4. H. M. F. Andreotti, S. Zaunseder, Improved heart rate detection for camera-based photoplethysmography by means of Kalman filtering, (2015)

5. R. Brown, R. Frayne, Conf. Proc. IEEE Eng. Med. Biol. Soc 2008, 2586 (2008)

6. J. L. Z. Yue, Y. Shen, Time-frequency analysis of heart sounds in telemedicine consulting system for auscultation, (2012)

7. E. N. A. Dliou, E. Nationale, Arrhythmia ECG Signal Analysis using Non Parametric TimeFrequency Techniques, (2012) 
8. C. Z. N. Zhang, Y. Li, Conf. Comput. Sci. Serv. Syst. CSSS pp. 1339-1342 (2012)

9. H. Ali, Heart Sound Signal Modeling and Segmentation based on Improved Shannon Energy Envelogram using ..., (2007)

10. E. F. Gomes, E. Pereira, Aistats 1, 1 (2012)

11. S. S. M. A. Castro, M.T. Coimbra, Proc. Annu. Int. Conf. IEEE Eng. Med. Biol. Soc. EMBS 2016, 5388 (2020)

12. A. C. J. Pedrosa, T.T.V. Vinhoza, Int. Conf. IEEE Eng. Med. Biol. Soc. EMBC 2014, 2294 (2014)

13. S. S. M. A. Castro, M.T. Coimbra, Noninvasive Blood Pressure and the Second Heart Sound Analysis, (2014)

14. S. S. M. A. Castro, M.T. Coimbra, Proc. Annu. Int. Conf. IEEE Eng. Med. Biol. Soc. EMBS pp. 3909-3912 (2013)

15. M. F. M. ller, Neural Networks 6, 525 (1993) 